\title{
Longitudinal evaluation of quality of life in 288 patients with neurofibromatosis 2
}

\author{
Rosalie E. Ferner • Adam Shaw • D. Gareth Evans - Dympna McAleer • \\ Dorothy Halliday • Allyson Parry • F. Lucy Raymond · Juliette Durie-Gair • \\ C. Oliver Hanemann · Rachel Hornigold · Patrick Axon • John F. Golding
}

Received: 24 November 2013/Revised: 24 February 2014/Accepted: 25 February 2014/Published online: 12 March 2014

(C) The Author(s) 2014. This article is published with open access at Springerlink.com

\begin{abstract}
Advances in molecular biology have resulted in novel therapy for neurofibromatosis 2-related (NF2) tumours, highlighting the need for robust outcome measures. The disease-focused NF2 impact on quality of life (NFTI-QOL) patient questionnaire was assessed as an outcome measure for treatment in a multi-centre study. NFTI-QOL was related to clinician-rated severity (Clin$\mathrm{Sev})$ and genetic severity (GenSev) over repeated visits. Data were evaluated for 288 NF2 patients ( $n=464$ visits) attending the English national NF2 clinics from 2010 to 2012. The male-to-female ratio was equal and the mean age was 42.2 (SD 17.8) years. The analysis included NFTI-
\end{abstract}

R. E. Ferner - A. Shaw - D. G. Evans - D. McAleer ·

D. Halliday - A. Parry - F. L. Raymond - J. Durie-Gair .

C. O. Hanemann · R. Hornigold · P. Axon · J. F. Golding

National Neurofibromatosis 2 Service, England, UK

R. E. Ferner $(\bowtie) \cdot R$. Hornigold

Department of Neurology, Guy's Hospital, Guy's and St.

Thomas' NHS Foundation Trust, Great Maze Pond,

London SE1 9RT, UK

e-mail: rosalie.ferner@kcl.ac.uk

R. E. Ferner

Department of Clinical Neuroscience, Institute of Psychiatry,

Kings College London, London, UK

A. Shaw

Department of Genetics, Guy's and St. Thomas' NHS

Foundation Trust, London, UK

D. G. Evans · D. McAleer

Department of Medical Genetics, Central Manchester University

Hospitals Foundation Trust, Manchester, UK

D. Halliday

Department of Clinical Genetics, Oxford Radcliffe Hospitals

NHS Trust, Oxford, UK
QOL eight-item score, ClinSev graded as mild, moderate, or severe, and GenSev as a rank order of the number of NF2 mutations (graded as mild, moderate, severe). The mean (SD) 8.7 (5.4) score for NFTI-QOL for either a first visit or all visits 9.2 (5.4) was similar to the published norm of 9.4 (5.5), with no significant relationships with age or gender. NFTI-QOL internal reliability was good, with a Cronbach's alpha score of 0.85 and test re-test reliability $r=0.84$. NFTI related to ClinSev $(r=0.41, p<0.001$; $r=0.46$ for all visits), but weakly to GenSev ( $r=0.16$, $p<0.05 ; r=0.15$ for all visits). ClinSev related to GenSev $(r=0.41, p<0.001 ; r=0.42$ for all visits). NFTIQOL showed a good reliability and ability to detect

A. Parry

Department of Neurology, Oxford Radcliffe Hospitals NHS

Trust, Oxford, UK

F. L. Raymond

Department of Medical Genetics, Addenbrooke's Hospital, University of Cambridge, Cambridge University Hospitals NHS Foundation Trust, Cambridge, UK

J. Durie-Gair · P. Axon

Department of Skull Base Surgery, Addenbrooke's Hospital, Cambridge University Hospitals NHS Foundation Trust, Cambridge, UK

C. O. Hanemann

Centre for Biomedical Research, Peninsula Schools of Medicine and Dentistry, Plymouth University, Plymouth, UK

J. F. Golding

Department of Psychology, University of Westminster, London, UK 
significant longitudinal changes in the QOL of individuals. The moderate relationships of NFTI-QOL with clinicianand genetic-rated severity suggest that NFTI-QOL taps into NF2 patient experiences that are not encompassed by ClinSev rating or genotype.

Keywords Neurofibromatosis $2 \cdot$ NF2 - NFTI-QOL . Vestibular schwannoma

\section{Introduction}

Neurofibromatosis 2 (NF2) is an inherited tumour suppressor disease with a prevalence of 1 in 60,000 and a birth incidence of 1 in 25-33,000 individuals [1, 2]. Bilateral vestibular schwannomas (VS) are emblematic of NF2, but schwannomas may form on other cranial, spinal, and peripheral nerves. Central nervous system meningiomas and ependymomas, peripheral neuropathy, amyotrophy, retinal hamartomas, and subcapsular lens opacities are part of the NF2 disease spectrum [1,3]. Typically the presenting symptoms in adults are hearing loss and balance disturbance, and these symptoms reflect the major causes of morbidity and impact on quality of life (QOL) in people with NF2 [1, 3, 4]. Impaired vision and facial weakness may compound the problems in individuals who already have to contend with deafness. The NF2 gene was identified on chromosome 22q11.2 and somatic mosaicism is present in about one-third of de novo patients $[1,2,5]$. Germline truncating mutations are associated with more severe disease than large deletions and missense mutations; individuals with germline truncating mutations are diagnosed at a younger age and usually have an earlier onset of symptomatic tumours [6, 7]. Recent developments in neurosurgery, radio-surgery, auditory rehabilitation, and molecular biology have increased the treatment options for individuals with NF2 [8]. Bevazicumab acts as a vascular endothelial growth factor inhibitor that reduces vestibular schwannoma growth and shrinks tumours in some patients; other novel drugs are being investigated in clinical trials, including lapatinib, a tyrosine kinase inhibitor, RAD0001 [9-11]. However, randomised controlled therapeutic trials, the gold standard of efficacy, are difficult to undertake because of the rarity of NF2. Assessment of genotype, in combination with clinician- and patient-rated severity is useful to determine therapeutic outcomes. Meticulous clinical evaluation includes neurological and visual examination, timed gait assessment, neurophysiology, speech and pure tone audiometry, and serial, standardised measurement of tumours on 1-mm magnetic resonance imaging $[1,8]$. Few studies have addressed QOL in people with NF2. Neary et al. [12] reported that the predominant problems were impaired balance and difficulty with social communication when they used a closed-set questionnaire and the generic Short Form-36 (SF-36) questionnaire to evaluate QOL in NF2 patients $[12,13]$. Patel undertook semi-structured interviews in six patients and revealed that NF2 had a negative impact on daily activities including employment, and was associated with social isolation arising from communication difficulties [4]. Family played a central role in providing physical, psychological, and emotional support. We developed the NF2 Impact on Quality of Life (NFTI-QOL) questionnaire, a reliable, validated disease-focused assessment for NF2 patients, for health-care providers to use as a clinical assessment tool and outcome measure (Table 1) [14]. The questionnaire is completed in a few minutes and comprises eight questions, with a maximum score of 24 reflecting the greatest impact on QOL. There is a free-response section at the end if individuals wish to add expand an answer and add new information. NFTI-QOL covers the domains of balance and dizziness, hearing, facial weakness, sight, mobility and walking, role and outlook on life, pain, anxiety, and depression [14] (Table 1).

\begin{abstract}
Aims
The aim of this study was to evaluate the NFTI-QOL as an assessment tool and potential outcome measure for therapeutic intervention in multi-centre national NF2 clinical services. Patient-reported experience using the NFTI-QOL was related to clinician-rated and genetic severity (GenSev) scores. Changes over repeated clinic visits were evaluated to detect stable disease and changes after intervention.
\end{abstract}

\section{Methods}

Ethical approval

The study was approved as a clinical evaluation, with study number 3711, by the Clinical Audit Group committee at Guy's and St Thomas' National Health Service (NHS) Foundation Trust, London.

Participating centres, inclusion and exclusion criteria

NIFTI-QOL data were collected for all patient visits to the national NF2 services at Guy's and St. Thomas' NHS Foundation Trust, Oxford University Hospitals NHS Trust, Addenbrooke's Hospital, Cambridge University NHS Foundation Trust, Central Manchester University Hospitals Foundation Trust, and satellite NF2 services from April 
Table 1 NFTI-QOL English version for the UK (neurofibromatosis 2 impact on quality of life) (reproduced with permission from Hornigold et al. [14])

INSTRUCTIONS FOR COMPLETING THE NFTI-QOL

Please complete the following information:

Age: years

Gender: Male $\square$ Female $\square$ (please tick)

For each of the questions on the next page, please tick the one box that describes how you feel today Usual activities include: work; housework; study; sport; social; family or leisure activities

Q1. Do balance or dizziness problems stop you performing your usual activities?

No balance problems or dizziness

Balance or dizziness problems but no difficulties

Balance or dizziness problems cause me some difficulties

Balance or dizziness problems stop my usual activities $\quad \square 3$

Q2. Do hearing problems stop you performing your usual activities?

No hearing problems

Hearing problems but no difficulty

Hearing problems cause me some difficulty

Hearing problems stop my usual activities

$\square 0$

$\square 1$

$\square 0$

$\square 1$

$\square 2$

$\square 3$

Q3. Does facial weakness stop you performing your usual activities?

No facial weakness

Facial weakness, but no difficulty

Facial weakness causes some difficulty

Facial weakness stops my usual activities

$\square 0$

$\square 1$

$\square 2$

$\square 3$

Q4. Do problems with your sight stop you performing your usual activities?

No problems with sight

Sight problems, but no difficulty

Sight problems cause me some difficulty

Sight problems stop my usual activities

Q5. Do you have any problems in mobility and walking?

No problems in mobility and walking

Some difficulty but can manage on my own

Unable to walk around without some help

Unable to walk at all

Q6. Has your medical condition affected your role and outlook on life?

(E.g. confidence, vulnerability, relationships, caring for family, career, having children)

No effect or positive effect

Small negative effect

Moderately negative effect

Large negative effect

$$
\square 0
$$

$\square 1$

$\square 2$

$\square 3$

$\square 0$

$\square 1$

$\square 2$

$\square 3$

$\square 1$

$\square 2$

$\square 3$

Q7. Pain; throughout our lives, most of us have had pain from time to time such as mild headaches, sprains and toothaches. Have you had pain other than this in the last week?

None

Mild pain

Moderate pain

Severe pain

Q8. Do you currently suffer from anxiety or depression?

No

Mild anxiety or depression

$$
\begin{aligned}
& \square 0 \\
& \square 1 \\
& \square 2 \\
& \square 3
\end{aligned}
$$

Moderate anxiety or depression

Extreme anxiety or depression

$\square 0$

$\square 1$

$\square 2$

$\square 3$

If you have any further comments regarding the impact of NF2 on your quality of life, please write them here:

You have now completed the NFTI-QOL. Thank you for your input.

2010 to October 2012. Individuals who did not fulfil the diagnostic criteria for generalised or mosaic NF2, or who were aged $<16$ years were excluded from the study. Age and gender were recorded for all patients.
Clinician-rated severity

Clinician-rated severity was assessed as severe, moderate, or mild and was determined during the clinical visit. Severe 
disease was classified as symptomatic presentation at age $<20$ years with at least two symptomatic or large tumours $>1.5 \mathrm{~cm}$, in addition to $\mathrm{VS}$ and including tumours removed previously. Individuals who were diagnosed with a central nervous system tumour before the age of 12 years and had at least one other symptomatic tumour were also rated as severe. Patients older than 30 years at presentation with no more than two symptomatic or large tumours $>1.5 \mathrm{~cm}$ including VS and tumours excised previously were assessed as mild. Moderate disease was classified as not meeting mild or severe criteria $[1,3]$.

Genetic-rated severity

Genetic severity was rated as severe, moderate, or mild. Truncating mutations in exons 1-13 in all cells were rated as severe. Moderate disease included (1) deletion not involving the promoter region or exon 1, (2) splice site mutations in exons 1-8, and (3) mosaicism of truncating mutations in exons $1-13$ in blood. Mild disease was classified as (1) a missense mutation or an inframe deletion, (2) a large deletion involving the promoter region or exon 1 , (3) a splice-site mutation in exons 9-15, (4) mosaicism (excluding moderate criteria), and (5) no mutation identified on blood analysis [1, 6, 7, 15, 17-19].

\section{Clinical assessment and NFTI-QOL}

At each clinic visit, patients underwent clinical assessment and completed the NFTI-QOL, an eight-item questionnaire (and a free-response section) with a maximum score of 3 per item (four-point scale, range 0-3, with three as the most impaired) and total score of 24 [14]. Patients who were unable to complete the questionnaire due to visual or motor difficulties were assisted by a specialist nurse.

\section{Statistics}

The NFTI-QOL, clinical, and genetic data were analysed with SPSS using correlation, linear regression, and analysis of variance (ANOVA) tools as required.

\section{Results}

Patient and visit numbers

Data were evaluated for 288 NF2 patients attending the national NF2 clinics and satellite centres in England (London, Oxford, Cambridge, Manchester) from 2010 to 2012. In total, there were 464 patient visits, including 288 $(62.1 \%)$ attending one visit, $117(25 \%)$ attending two visits, $41(8.9 \%)$ with three visits, 14 (3\%) with four visits, two patients $(0.4 \%)$ having five visits, and two $(0.4 \%)$ attending six visits.

Gender, age, clinical- and genetic-rated severity

The studied group was comprised of 143 males and 145 females; the mean age was 42.2 years (SD 17.8) and the age range was 16-87 years. There were no significant differences in age or gender of the patients attending the four different NF2 centres. Clinical- and genetic-rated severity for 288 individuals with NF2 is shown in Table 2. Thirty patients elected not to have genetic testing. There were no significant differences in genetic-rated severity between the four centres, but mean clinical severity (ClinSev) was milder in the Manchester centre (1.5) than in London (2.0), Cambridge (2.0), or Oxford (1.9) (Kruskal Wallace test, $\left.d f 3, \chi^{2} 24.8, p<0.01\right)$.

\section{NFTI-QOL}

The mean NFTI-QOL score for 288 patients for the first clinic visit was 8.7 (SD 5.4), for the 117 patients who attended on a second visit it was 9.3 (SD 5.2), and for all visits the mean NFTI-QOL was 9.2 (SD 5.4). This was similar to the published norm of 9.4 (5.5), with no significant relationships with age or gender. Analysis of variance (ANOVA) revealed no differences in NFTIQOL scores for the four NF2 sites. Hearing loss, dizziness and balance, and impact of NF2 on role and outlook on life were the items that showed the highest severity ratings for all centres (Fig. 1). The evaluation of patientrated QOL (London) from visit 1 to visit 2 showed that the majority of patients had stable disease. However, three patients were significantly worse and three showed significant improvement, as demonstrated by the $95 \%$ confidence intervals (CIs) for individuals with NF2 (Fig. 2). Examination of longitudinal changes indicated that any NFTI-QOL score change up or down of greater than five points would be statistically significant for an individual (Fig. 2). The internal reliability of NFTI-QOL was found to be very good, with a Cronbach's alpha score of 0.85 and re-test reliability $r=0.84$. The NFTIQOL was related to ClinSev $(r=0.41, p<0.001$;

Table 2 Clinical- and genetic-rated severity in 288 NF2 patients

\begin{tabular}{lll}
\hline Severity & Clinical severity, $n(\%)$ & Genetic severity, $n(\%)$ \\
\hline Mild & $124(43.1)$ & $168(58.4)$ \\
Moderate & $93(32.3)$ & $49(17)$ \\
Severe & $70(24.3)$ & $41(14.2)$ \\
Not tested & 0 & $30(10.4)$ \\
Not classified & $1(0.3)$ & $0(0)$ \\
\hline
\end{tabular}




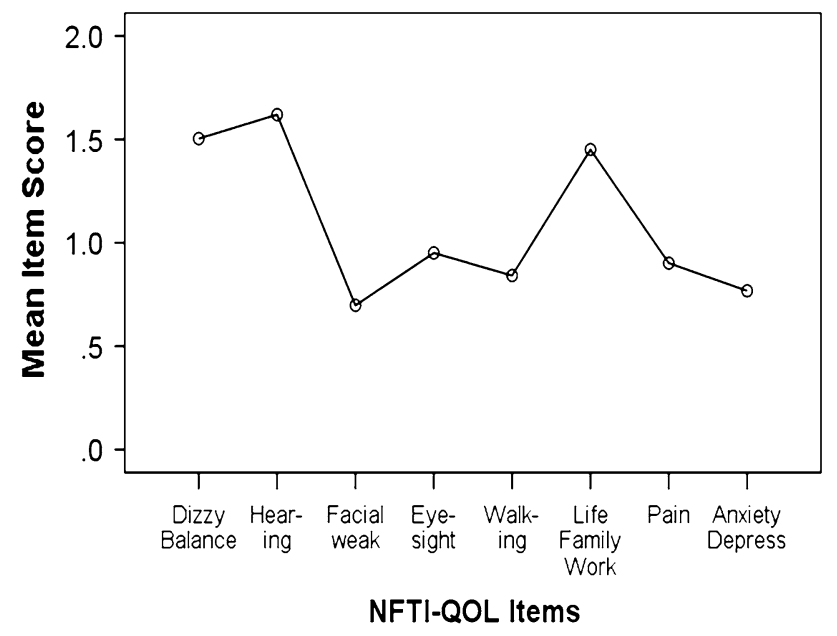

Fig. 1 Mean item scores for NFTI-QOL in 288 patients on first visit to London, Oxford, Cambridge, or Manchester NF2 centre

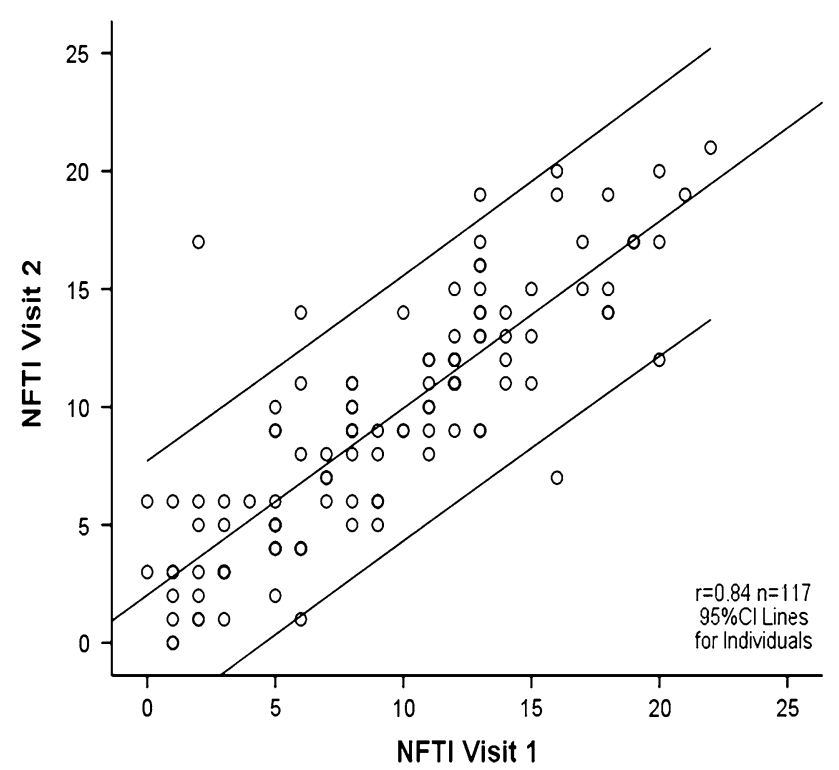

Fig. 2 NFTI-QOL scores are shown for NF2 individuals who attended both visit 1 and visit $2(n=117)$. The thick line is the fitted regression line and the thin lines on either side denote a $95 \% \mathrm{CI}$ for individuals. The darker circles indicate superimposed observations for multiple NF2 individuals

$r=0.46$ for all visits), but was only related weakly to GenSev ( $r=0.16, p<0.05 ; r=0.15$ for all visits). Clinical severity in turn related to GenSev $(r=0.41$, $p<0.001 ; r=0.42$ for all visits).

\section{NFTI-QOL free responses}

There were infrequent free responses, and where present, they tended to amplify one of the eight NFTI-QOL domains, rather than report new symptoms or problems.
One individual rated all domains as 0 on the first clinic visit, despite complaining of worsening hearing, balance, and role and outlook on life in the clinic. On the second visit, staff found that the patient had not learnt to read at school and that the total NFTI-QOL score was actually 6 when appropriate assistance was given for completion of the questionnaire.

\section{Discussion}

In this 2-year longitudinal study, we assessed clinical-, genetic-, and patient-rated disease severity in 288 patients in four NF2 centres in England. We evaluated the mean total and individual item NFTI-QOL scores for the four NF2 centres. This study did not reveal any significant difference in the mean NFTI-QOL or individual item scores. NFTIQOL showed good internal reliability, good test-retest reliability and demonstrated stability over time. Moreover, the mean NFTI-QOL and individual item scores remained stable for multiple visits, with no particular trend for increase or decrease in items over time. Obviously, there were wide variations in NFTI-QOL scores between individuals, reflecting different perceptions and coping mechanisms in response to chronic disease. Within the context of the overall high reliability and stability of the NFTI-QOL, an increase or reduction in scores was evident in some individuals over time. The total NFTI-QOL score is the optimum measure for estimating an individual's overall QOL and changes that occur over time. The clinician may find it useful to evaluate the individual item scores to look for changes in a particular domain, as this reflects the relative importance of that domain to the individual. Scores vary with individual circumstances and ability of that individual to cope with the impact of the disease on that particular domain. For example, a 32-year-old male had deteriorating total NFTI-QOL scores (from 13 to 16) until a cochlear implant was inserted and specialist neuro-rehabilitation was undertaken for impaired mobility. The total NFTI-QOL score reduced to 9 and individual item scores improved for hearing, balance, mobility, and role and outlook on life. This indicates the potential for NFTI-QOL to detect changes in disease symptoms or response to treatment. It also underlines the observation that improvement in one domain, for instance hearing, may generalise to improvements in other domains. There was a change of more than five points in the total NFTI-QOL score in five other patients, including four males and one female, aged 19-62 years (median age 38 years). The total NFTI-QOL scores decreased in three individuals due to improved hearing and balance and neurological symptoms. The total NFTI-QOL scores increased in two patients with worsening hearing, balance, and psychological issues that had a 
deleterious impact on their role and outlook in life. A fivepoint change or greater was statistically significant for an individual (95\% CI for individuals). However, this is a statistical cutoff and smaller changes in scores could well have clinical significance for an individual (Fig. 2). Although NFTI-QOL correlates with ClinSev, it appears to tap into more about the impact of NF2 on the individual, rather than simply being a reflection of clinical and GenSev. Overall, ClinSev was rated as milder for patients attending the Manchester centre compared with London, Cambridge, and Oxford, and this might simply reflect the disease heterogeneity. There was a weak correlation between NFTIQOL and GenSev. This could have arisen because GenSev looks at the disease from a long-term perspective, whereas the patient-rated NFTI-QOL mirrors current disease status. The weaker correlation between NFTI-QOL and GenSev might have arisen because some individuals with a mutation predicting severe NF2 were assessed early in the course of their disease and had not yet developed many symptoms or deficits. Furthermore, individuals with genetic mutations associated with mild disease, may still have significant clinical symptoms and neurological deficit, albeit with a lower tumour burden and older age at presentation. One might expect that anxiety and depression would be a major feature of NF2, and this is the case for some. However, the mean item score for anxiety and depression for all visits was low, and this likely reflects the high levels of psychological, psychiatric, and educational support available to people attending the national NF2 centres. Most patients did not have visual problems and the mean NFTI-QOL score for sight was low, but it was reported as a major problem for a minority of individuals particularly when it compounded hearing loss. Disease specific QOL questionnaires are an important tool for evaluating patient focused outcomes of intervention. If a new agent is capable of shrinking a tumour but the patient does not experience an improvement in symptoms or QOL, careful thought should be put into continuing administration of the drug, unless it reduces mortality rates. One of our patients had a significant reduction in size of a VS following 3 months of treatment with bevacizumab; nonetheless, the total NFTI-QOL score remained 22, as the patient had not noted improvement in any of the NFTI-QOL domains. NFTI-QOL is validated for people with NF2 16 years and older, but potentially could be extended for use in 12-year-olds. The cohort of younger children with NF2 is small, and current clinical trials are focused on teenagers and adults. Further work would be required to develop a disease-specific NF2 QOL questionnaire in this younger age group. This is because visual impairment, neurological deficit from amyotrophy, central nervous system ependymomas, and meningiomas may be more prominent findings than hearing, loss of balance, and dizziness from VS $[1,3]$.

\section{Conclusions}

NFTI-QOL is a disease-specific questionnaire that is quick and easy to administer. It shows good reliability and has the ability to detect significant changes over time in QOL of individual patients. We have demonstrated previously that NFTI-QOL correlates strongly and significantly with all domains of the SF-36 and with the EuroQOL, generic questionnaires that do not focus on NF2-specific problems $[13,16]$. The moderate relationship between NFTI-QOL and ClinSev and genetic-rated severity was consistent with the notion that NFTI-QOL draws on other dimensions of NF2 patient experiences that are not covered by either of these latter measures.

Acknowledgments We are grateful to all clinicians in the national NF2 centres and satellite units for their clinical input. We would like to thank the NF2 nurses for assisting patients in the clinic. We acknowledge the help of Vilka Scott-Kitching, Steve Martin, Andrew McCarthy, and Sally Maspero in data entry. We are very grateful to the national commissioning team for funding the NF2 services in England and to our NF2 patients. The national NF2 services were funded by the national specialist commissioning team in April 2010.

Conflicts of interest On behalf of all authors, the corresponding author states that there are no conflicts of interest.

Open Access This article is distributed under the terms of the Creative Commons Attribution License which permits any use, distribution, and reproduction in any medium, provided the original author(s) and the source are credited.

\section{References}

1. Evans DG (2009) Neurofibromatosis type 2 (NF2): a clinical and molecular review. Orphanet J Rare Dis 4:16

2. Evans DGR, Howard E, Giblin C, Clancy T, Spencer H, Huson SM, Lalloo F (2010) Birth incidence and prevalence of tumour prone syndromes: estimates from a UK genetic family register service. Am J Med Genet A 152A(2):327-332

3. Ferner R (2007) Neurofibromatosis 1 and neurofibromatosis 2: a twenty-first century perspective. Lancet Neurol 6(4):340-345

4. Patel CM, Ferner R, Grunfeld EA (2011) A qualitative study of the impact of living with neurofibromatosis type 2. Psychol Health Med 16(1):19-28

5. Rouleau GA, Merel P, Lutchman M, Sanson M, Zucman J, Marineau C, Hoang-Xuan K, Demczuk S, Desmaze C, Plougastel B, Pulst SM, Lenoir G, Bijlsma E, Fashold R, Dumanski J, De Jong P, Parry D, Eldridge R, Aurias A, Delattre O, Thomas G (1993) Alteration in a new gene encoding a putative membraneorganizing protein causes neuro-fibromatosis type 2. Nature 363(6429):515-521

6. Ruttledge MH, Andermann AA, Phelan CM, Claudio JO, Han FY, Chretien N, Rangaratnam S, MacCollin M, Short P, Parry D, Michels V, Riccardi VM, Weksberg R, Kitamura K, Bradburn JM, Hall BD, Propping P, Rouleau GA (1996) Type of mutation in the neurofibromatosis type 2 gene (NF2) frequently determines severity of disease. Am J Hum Genet 59(2):331-427

7. Evans DGR, Trueman L, Wallace A, Collins S, Strachan T (1998) Genotype/phenotype correlations in type 2 neurofibromatosis: 
evidence for more severe disease with truncating mutations. J Med Genet 35(6):450-455

8. Blakeley JO, Evans DG, Adler J, Brackmann D, Chen R, Ferner RE, Hanemann CO, Harris G, Huson SM, Jacob A, Kalamarides M, Karajannis MA, Korf BR, Mautner VF, McClatchey AI, Miao H, Plotkin SR, Slattery W 3rd, Stemmer-Rachamimov AO, Welling DB, Wen PY, Widemann B, Hunter-Schaedle K, Giovannini M (2012) Consensus recommendations for current treatments and accelerating clinical trials for patients with neurofibromatosis type 2. Am J Med Genet A 158A(1):24-41

9. Plotkin SR, Stemmer-Rachamimov AO, Barker FG, Halpin C, Padera TP, Tyrrell A, Sorensen AG, Jain RK, di Tomaso E (2009) Hearing improvement after bevacizumab in patients with neurofibromatosis type 2. N Eng J Med 361(14):358-367

10. Plotkin SR, Merker VL, Halpin C, Jennings D, McKenna MJ, Harris GJ, Barker FG 2nd (2012) Bevacizumab for progressive vestibular schwannoma in neurofibromatosis type 2: a retrospective review of 31 patients. Otol Neurotol 33(6):1046-1052

11. Karajannis MA, Legault G, Hagiwara M, Ballas MS, Brown K, Nusbaum AO, Hochman T, Goldberg JD, Koch KM, Golfinos JG, Roland JT, Allen JC (2012) Phase II trial of lapatinib in adult and paediatric patients with neurofibromatosis type 2 and progressive vestibular schwannomas. Neurol Oncol 14(9):1163-1170

12. Neary WJ, Hillier VF, Flute T, Stephens SD, Ramsden RT, Evans DG (2010) The relationship between patients' perception of the effects of neurofibromatosis type 2 and the domains of the short form-36. Clin Otolaryngol 35(4):291-299

13. Ware JE, Snow KK, Kosinski M, Gandek B (1993) SF-36 health survey. Manual and interpretation guide. The Health Institute, Boston
14. Hornigold RE, Golding JF, Leschziner G, Obholzer R, Gleeson MJ, Thomas N, Walsh D, Saeed S, Ferner RE (2012) The NFTIQOL: a disease specific quality of life questionnaire for neurofibromatosis 2. J Neurol Surg Part B Skull Base 273(2):104-111

15. Baser ME, Kuramoto L, Woods R, Joe H, Friedman JM, Wallace AJ, Ramsden RT, Olschwang S, Bijlsma E, Kalamarides M, Papi L, Kato R, Carroll J, Lázaro C, Joncourt F, Parry DM, Rouleau GA, Evans DG (2005) The location of constitutional neurofibromatosis 2 (NF2) splice site mutations is associated with the severity of NF2. J Med Genet 42:540-546

16. The Euroqol Group (1990) Euroqol-a new facility for the measurement of health-related quality of life. Health Policy 16:199-208

17. Selvanathan SK, Shenton A, Ferner R, Wallace AJ, Huson SM, Ramsden RT, Evans DG (2010) Further genotype-phenotype correlations in neurofibromatosis type 2. Clin Genet 77(2): $163-170$

18. Smith MJ, Higgs JE, Bowers NL, Halliday D, Paterson J, Gillespie J, Huson SM, Freeman SR, Lloyd S, Rutherford SA, King AT, Wallace AJ, Ramsden RT, Evans DG (2011) Cranial meningiomas in 411 neurofibromatosis type 2 (NF2) patients with proven gene mutations: clear positional effect of mutations, but absence of female severity effect on age at onset. J Med Genet 48(4):261-265

19. Evans D, Bowers N, Huson S, Wallace A (2013) Mutation type and position varies between mosaic and inherited NF2 and correlates with disease severity. Clin Genet 83:594-595 